\title{
Central Nervous System: Notable Developments in the Management of Primary and Recurrent Gliomas
}

\author{
Presented by Louis Burt Nabors, MD
}

\begin{abstract}
Over the past 30 years, the incidence of primary brain tumors has been increasing. Primary brain tumors are a heterogeneous group of central nervous system cancers with a wide range of outcomes and therapeutic strategies. As a result, prognostic features and molecular markers play a critical role in selecting treatment options for patients with brain tumors. At the NCCN 21st Annual Conference, Louis Burt Nabors, MD, reviewed the most appropriate adjuvant therapy for 3 types of brain tumors-primary low-grade gliomas, anaplastic oligodendrogliomas, and malignant gliomas-and shared supportive clinical trial data and NCCN recommendations.
\end{abstract}

J Natl Compr Canc Netw 2016;14(5.5):681-684

Management of adult central nervous system (CNS) cancers represents a continually evolving field, with published data from clinical trials serving as the foundation for appropriate treatment decisions. Emerging and ongoing studies should shed more light on the current roles of adjuvant radiotherapy (RT) and chemotherapy (and their optimal sequencing) and on the importance of incorporating prognostic information into the mix.

\section{Primary Low-Grade Gliomas}

Although gliomas represent a small percentage of all cancers, they cast a big shadow in regard to measure of disease burden on patients, and the related public health cost of the disease and its treatment in the United States is among the highest in oncology, revealed Louis Burt Nabors, MD, Vice-Chairman of Neurology, Professor, and Director of the Neuro-Oncology Program at the University of Alabama at Birmingham Comprehensive

Presented by Louis Burt Nabors, MD, Department of Neurology, Division of Neuro-Oncology, University of Alabama at Birmingham, Birmingham, Alabama.

Dr. Nabors has disclosed that he is a scientific advisor for Cavion and Cortice Biosciences, Inc.; and that he receives other financial benefit from Ziopharm Oncology, Inc.

Correspondence: Louis Burt Nabors, MD, Department of Neurology, Division of Neuro-Oncology, University of Alabama at Birmingham, 510 20th Street South, FOT 1020, Birmingham, AL 35294.

E-mail: bnabors@uab.edu
Cancer Center. "Although [primary gliomas] are called low grade, they are not benign," cautioned Dr. Nabors, who is also Chair of the NCCN CNS Cancers Panel. "Their goal is to transform into a more malignant and aggressive tumor type."

Dr. Nabors shared a case study to illustrate how evaluation of prognostic signs can assist in making treatment decisions. A 31-year-old man with a remote history of concussion experienced a new-onset partial seizure. Workup included a normal neurologic examination, and results of an MRI revealed glioma. Surgery was performed and a very clean resection was achieved.

Based on the 2015 NCCN Guidelines for CNS Cancers, the optimal treatment recommendation for this patient was observation, and Dr. Nabors summarized both the favorable and unfavorable prognostic signs to support this course of action. Good prognostic signs include age younger than 40 years, seizures at presentation, absence of neurologic deficits, and gross total resection-all of which the patient in the case study presented. Poor prognostic signs include a tumor diameter larger than 5 to 6 $\mathrm{cm}$ and the presence of contrast enhancement.

He then reviewed some of the supportive data behind the treatment options for those with high-risk gliomas. Among the treatment options for diffuse astrocytomas (low-grade gliomas) are surgery alone followed by RT, chemotherapy (with PCV [procarbazine, lomustine, 
Nabors

and vincristine] or temozolomide), and chemoradiotherapy (chemoRT). Adjuvant chemoRT with PCV is a category 1 recommendation for patients with high-risk disease.

"Through the years, there has been some controversy regarding the timing of RT after surgery," admitted Dr. Nabors. "Some groups feel like it should begin after completion of and healing from surgery, and others feel like it is okay to wait until disease progression."

Supportive data for this category 1 recommendation are derived from the results of the RTOG 9802 trial. ${ }^{1,2}$ In the trial, patients were stratified into lowrisk and high-risk groups, with those at low risk undergoing observation. The high-risk group was randomized to receive either standard RT alone or RT plus PCV chemotherapy. Progression-free survival data showed "separation of the survival curves, with a nice reduction in the hazard ratio at 0.50 , favoring radiation followed by chemotherapy over radiation alone," said Dr. Nabors. Benefits in progression-free survival "heavily favored" the combination therapy versus RT alone. Benefits in overall survival (OS) also favored the combination arm (10-year rates of $60.1 \%$ vs $40.1 \%$ ). "This is actually one of the first prospective studies to show a treatment-related increase in survival for patients with low-grade glioma," announced Dr. Nabors. "It has been a hard group to study because of the heterogeneous clinical nature [of low-grade glioma]."

In the 2015 NCCN Guidelines, for patients with high-risk gliomas, RT and adjuvant and/or concurrent temozolomide are options, but they carry a category 2B designation. "These studies began in the 1990s, before temozolomide was available," reminded Dr. $\mathrm{Na}$ bors. "Studies are looking at this important question today, comparing PCV and temozolomide [Figure 1]."

Closing his discussion on low-grade gliomas, Dr. Nabors centered on the age criterion and the role of an experienced surgeon. The age of 40 years should be used as a general reference point, he advised. "There are 40-year-olds who appear a lot older than 40 , and there are 50-year-olds who appear a lot younger. So, I wouldn't get too fixated on the number as an absolute." With surgical resection playing a key role in the treatment of these patients, the skill level of the surgeon warrants attention. "I highly encourage [patients] to see a neurosurgeon experienced in the resection of glioma," he added.

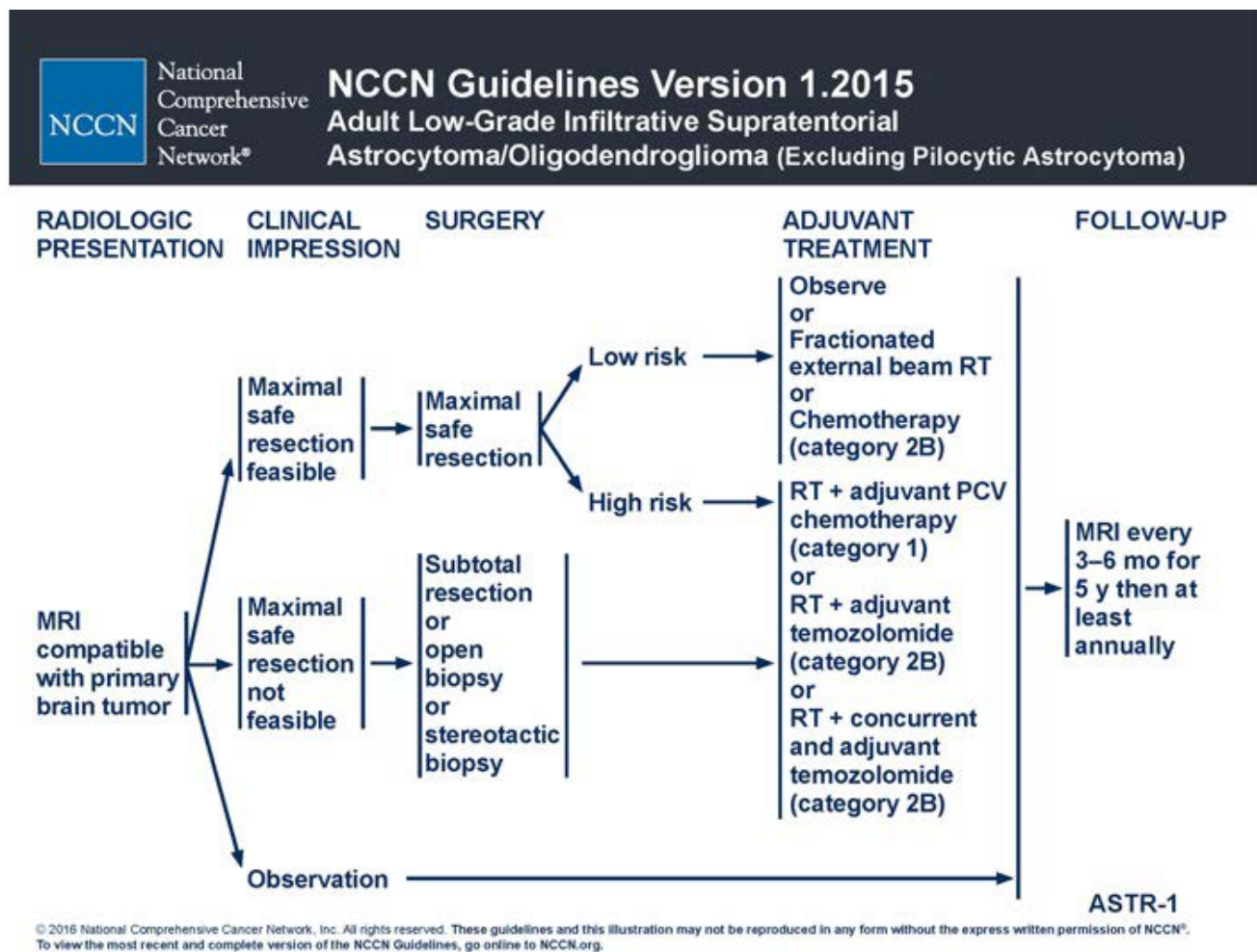

Figure 1. NCCN Guidelines for the treatment of adult low-grade infiltrative supratentorial astrocytoma/oligodendroglioma. 
Developments in Glioma Management

\section{Anaplastic Oligodendrogliomas}

Oligodendrogliomas are classified by the WHO as a grade III neoplasm and have a distinct histologic appearance. In addition, the combined allelic loss of chromosomes $1 \mathrm{p}$ and $19 \mathrm{q}$ is found in approximately $60 \%$ to $70 \%$ of anaplastic tumors. Brain tumors with these type of chromosomal abnormalities have been associated with longer survival and greater chemosensitivity, shared Dr. Nabors.

Optimal treatment recommendations for anaplastic oligodendrogliomas consist of RT followed by PCV. Dr. Nabors briefly reviewed the data supporting these options, focusing on 2 studies in newly diagnosed disease: RTOG $9402^{3,4}$ and EORTC 26951.5 The trial design for these studies was slightly different: for RTOG 9402, neoadjuvant therapy consisted of PCV chemotherapy before RT, and in EORTC 26951 adjuvant therapy consisted of PCV chemotherapy after RT. However, toxicity from the PCV regimen was an issue in both studies. "A lot of patients did not complete all of the prescribed cycles of chemotherapy," revealed Dr. Nabors.

Based on the findings of both RTOG 9402 and EORTC 26951, the adjuvant treatment pathway in the 2015 NCCN Guidelines for CNS Cancers hinges on the pathology results of the anaplastic gliomas. In both trials, patients who lacked $1 p$ and $19 q$ alleles tended to derive more benefit from PCV chemotherapy and RT than from RT alone compared with those who had non-1p/19-deleted tumors. ${ }^{3-5}$ There was a substantial reduction in the hazard ratio, noted Dr. Nabors, "and almost a doubling of the overall median survival."

Thus, for the subset of patients with $1 p 19 q$ co-deleted tumors, fractionated external-beam RT (EBRT) and neoadjuvant or adjuvant PCV chemotherapy is a category 1 recommendation, with EBRT and temozolomide or PCV chemotherapy or temozolomide carrying category $2 \mathrm{~B}$ designations. For those with $1 \mathrm{p} 19 \mathrm{q}$ unideleted or nondeleted tumors, the category 1 recommendation in adjuvant treatment is fractionated EBRT.

However, the optimal treatment paradigm has not been established. Questions regarding the proper sequencing of these therapies and the comparative effectiveness of temozolomide versus PCV remain. Currently, 2 ongoing cooperative group studies are attempting to answer the question on temozolomide compared with PCV.

\section{Malignant Gliomas}

Malignant gliomas can be categorized into anaplastic astrocytomas and glioblastoma multiforme (GBM); astrocytomas are less aggressive than glioblastomas. GBM is the most difficult primary brain tumor to treat, and "pathologically, it holds all the features of bad behavior," said Dr. Nabors.

Optimal treatment for malignant gliomas is generally temozolomide and RT. Supportive data for this therapy come from the randomized phase III EORTC 26981/22981-NCIC CE3 trial, which included nearly 600 patients with newly diagnosed resected glioblastoma from 85 centers. ${ }^{6}$ The study investigators found clinically meaningful benefit with the combination therapy over RT alone, although OS improved only by approximately 2.5 months and approximately $27 \%$ of patients were alive at 24-month follow-up.

A closer look at the therapeutic benefit revealed the role of inactivation of the DNA-repair enzyme methylguanine methyltransferase (MGMT). Hegi et $\mathrm{al}^{7}$ found that MGMT promoter was methylated in nearly half of the evaluable cases of glioblastoma. This was found to be an independent favorable prognostic factor-patients whose tumor contained a methylated MGMT promoter experienced a survival benefit, whereas those who did not have this promoter did not receive such a benefit. "We are increasingly going to have to become comfortable incorporating this predictive information into our practices," indicated Dr. Nabors. Several ongoing clinical trials are examining this issue.

Dr. Nabors briefly turned to a novel neurooncologic modality for antimitotic therapy known as tumor-treating fields. This continuous type of therapy, which is used with concomitant RT and temozolomide, requires that electrodes be placed on the shaved scalp based on the tumor's location and a battery pack carried for at least 18 hours each day. The phase III trial EF-14 demonstrated prolonged progression-free survival and OS with this regimen over chemotherapy alone in patients with newly diagnosed, resected glioblastoma. ${ }^{8}$ There was a 3-month difference in OS. 
Nabors

\section{References}

1. Buckner JC, Pugh SL, Shaw EG, et al. Phase III study of radiation therapy with or without procarbazine, CCNU, and vincristine in low-grade glioma: RTOG 9802 with Alliance, ECOG, and SWOG [abstract]. J Clin Oncol 2014;32(Suppl 5):Abstract 2000.

2. Shaw EG, Wang $M$, Coons $S W$, et al. Randomized trial of radiation therapy plus procarbazine, lomustine, and vincristine chemotherapy for supratentorial adult low-grade glioma: initial results of RTOG 9802. J Clin Oncol 2012;30:3065-3070.

3. Intergroup Radiation Therapy Oncology Group Trial 9402, Cairncross G, Berkey B, et al. Phase III trial of chemotherapy plus radiotherapy compared with radiotherapy al one for pure and mixed anaplastic oligodendroglioma: Intergroup Radiation Therapy Oncology Group trial 9402. J Clin Oncol 2006;24:2707-2714.
4. Cairncross $G$, Wang M, Shaw E, et al. Phase III trial of chemoradiotherapy for anaplastic oligodendroglioma: long-term results of RTOG 9402. J Clin Oncol 2013;31:337-343.

5. van den Bent MJ, Brandes AA, Taphoorn MJ, et al. Adjuvant procarbazine, lomustine, and vincristine chemotherapy in newly diagnosed anaplastic oligodendroglioma: long-term follow-up of EORTC brain tumor group study 26951. J Clin Oncol 2013;31:344-350.

6. Stupp R, Mason WP, van den Bent MJ, et al. Radiotherapy plus concomitant and adjuvant temozolomide for glioblastoma. N Engl J Med 2005;352:987-996.

7. Hegi ME, Diserens AC, Gorlia T, et al. MGMT gene silencing and benefit from temozolomide in glioblastoma. N Engl J Med 2005;352:997-1003.

8. Stupp R, Taillibert S, Kanner AA, et al. Maintenance therapy with tumortreating fields plus temozolomide vs temozolomide alone for glioblastoma: a randomized clinical trial. JAMA 2015;314:2535-2543. 